\title{
An atypical tension pneumothorax
}

\author{
Author: Shivasankar Murugan Sukumar ${ }^{\mathrm{A}}$
}

\section{Introduction}

Eosinophilic oesophagitis is a rare and chronic inflammatory disorder, with spontaneous oesophageal rupture reported in a handful of anecdotal case reports. We present an unusual case of delayed tension pneumothorax following occult oesophageal rupture, posing a significant diagnostic conundrum.

\section{Abstract}

A 29-year-old man with eosinophilic oesophagitis and asthma presented with chest pain and vomiting. Several hours later he developed loin to groin pain, pyrexia and renal angle tenderness. A urine dipstick was positive for blood and his inflammatory markers were significantly elevated. A chest X-ray (CXR) was unremarkable. Computed tomography (CT) urography excluded a calculus; however, it demonstrated left-sided consolidation with a small effusion. He was treated with antibiotics empirically. Over the next 48 hours, his inflammatory markers continued to rise. He developed sudden-onset dyspnoea and pleuritic chest pain associated with tachycardia and tachypnoea. A repeat CXR revealed a tension pneumothorax, requiring immediate needle decompression followed by chest drain insertion. This immediately drained $900 \mathrm{~mL}$ purulent fluid with a $\mathrm{pH}$ of 7.2. CT of the thorax revealed a gas and fluid collection in the mediastinum, possibly secondary to oesophageal rupture communicating with the pleural space. A barium swallow test demonstrated a leak at the gastroesophageal junction and he was transferred for surgical closure of the rupture.

\section{Discussion}

Although a tension pneumothorax can develop instantaneously at the time of oesophageal rupture, this case highlights the importance of considering oesophageal rupture as a cause of tension pneumothorax in any patient with a hydropneumothorax with persistent non-haemorrhagic drainage.

\section{Conflicts of interest}

None declared. 